\title{
Polynomial Kernels and Wideness Properties of Nowhere Dense Graph Classes*
}

\author{
Stephan Kreutzer \\ Technische Universität Berlin \\ stephan.kreutzer@tu-berlin.de
}

\author{
Roman Rabinovich \\ Technische Universität Berlin \\ roman.rabinovich@tu-berlin.de
}

\author{
Sebastian Siebertz \\ Technische Universität Berlin \\ sebastian.siebertz@tu-berlin.de
}

\begin{abstract}
Nowhere dense classes of graphs [24, 25] are very general classes of uniformly sparse graphs with several seemingly unrelated characterisations. From an algorithmic perspective, a characterisation of these classes in terms of uniform quasi-wideness, a concept originating in finite model theory, has proved to be particularly useful. Uniform quasi-wideness is used in many fpt-algorithms on nowhere dense classes. However, the existing constructions showing the equivalence of nowhere denseness and uniform quasi-wideness imply a non-elementary blow up in the parameter dependence of the fpt-algorithms, making them infeasible in practice. As a first main result of this paper, we use tools from logic, in particular from a sub-field of model theory known as stability theory, to establish polynomial bounds for the equivalence of nowhere denseness and uniform quasi-wideness.

A powerful method in parameterized complexity theory is to compute a problem kernel in a pre-computation step, that is, to reduce the input instance in polynomial time to a sub-instance of size bounded in the parameter only (independently of the input graph size). Our new tools allow us to obtain for every fixed radius $r \in \mathbb{N}$ a polynomial kernel for the distance- $r$ dominating set problem on nowhere dense classes of graphs. This result is particularly interesting, as it implies that for every class $\mathcal{C}$ of graphs which is closed under taking subgraphs, the distance- $r$ dominating set problem admits a kernel on $\mathcal{C}$ for every value of $r$ if, and only if, it already admits a polynomial kernel for every value of $r$ (under the standard assumption that FPT $\neq \mathrm{W}[2]$ ).
\end{abstract}

\section{Introduction}

Given a graph $G$ and an integer $k$, the Dominating SET problem is to determine the existence of a subset $D \subseteq V(G)$ of size at most $k$ such that every vertex $u \in V(G)$ is dominated by $D$, that is, if $u$ does not belong to $D$, then it must have a neighbour in $D$. The Dominating Set problem, parameterized by the size of the solution $k$, plays a central role in parameterized complexity theory, it is arguably one of the most important examples of a W[2]-complete problem, and hence considered intractable from the point of view of parameterized complexity on general graphs. A problem is

*This work has been supported by the European Research Council (ERC) under the European Union's Horizon 2020 research and innovation programme (ERC Consolidator Grant DISTRUCT, grant agreement No 648527). 
fixed-parameter tractable on a class $\mathcal{C}$ of graphs parameterized by the solution size $k$, if there is an algorithm deciding whether a graph $G \in \mathcal{C}$ admits a solution of size $k$ in time $f(k) \cdot|V(G)|^{c}$, for some computable function $f$ and constant $c$.

A particularly fruitful approach in parameterized complexity theory and algorithmic graph structure theory is the study of hard computational problems on restricted classes of inputs. This research is based on the observation that many problems such as Dominating SET, which are considered intractable in general, can be solved efficiently on classes of graphs such as graphs of bounded treewidth, planar graphs, or more generally, graph classes excluding a fixed minor.

An important goal of this line of research is to identify the most general classes of graphs on which a wide range of algorithmic problems can be solved efficiently. In this context, classes of graphs excluding a fixed minor have been studied intensively. More recently, even more general classes of graphs such as those excluding a fixed topological minor have received increased attention.

A useful method in parameterized complexity is to compute a problem kernel in a polynomial time pre-computation step, that is, to reduce the input instance to a sub-instance of size bounded in the parameter only (independently of the input graph size). The first important result of this type for the Dominating SET problem by Alber et al. [2] showed that there exists a kernel of linear size for the problem on planar graphs. Linear kernels were later found for bounded genus graphs [3], apex-minor-free graphs [13], $H$-minor-free graphs [14], and $H$-topological-minor-free graphs [15].

The algorithmic results on these graph classes are in one way or another based on topological arguments which can be derived from structure theorems for the corresponding class. Most notable structure theorems in this context are Robertson and Seymour's structure theorem for $H$-minor-free graphs [29] or its extension to $H$-topological-minor-free graphs by Grohe and Marx [18]. A complete shift in paradigm was initiated by Nešetřil and Ossona de Mendez with their ground-breaking work on bounded expansion [22, 23] and nowhere dense classes of graphs [24, 25]. On these classes, which properly extend the aforementioned classes defined by excluded (topological) minors, many topological arguments are replaced by much more general density based arguments.

Formally, a graph $H$ with $V(H)=\left\{v_{1}, \ldots, v_{n}\right\}$ is a minor of a graph $G$, written $H \preccurlyeq G$, if there are pairwise vertex disjoint connected subgraphs $H_{1}, \ldots, H_{n}$ of $G$ such that whenever $\left\{v_{i}, v_{j}\right\} \in$ $E(H)$, then there are $u_{i} \in V\left(H_{i}\right)$ and $u_{j} \in\left(H_{j}\right)$ with $\left\{u_{i}, u_{j}\right\} \in E(G)$. We call $\left(H_{1}, \ldots, H_{n}\right)$ a minor model of $H$ in $G$. The graph $H$ is a depth-r minor of $G$, denoted $H \preccurlyeq r G$, if there is a minor model $\left(H_{1}, \ldots, H_{n}\right)$ of $H$ in $G$ such that each $H_{i}$ has radius at most $r$. We denote the complete graph on $t$ vertices by $K_{t}$ and the complete bipartite graph with parts of size $s$ and $t$ by $K_{s, t}$.

Definition 1. A class $\mathcal{C}$ of graphs is nowhere dense if there is a function $f: \mathbb{N} \rightarrow \mathbb{N}$ such that $K_{f(r)} \aleph_{r} G$ for all $r \in \mathbb{N}$ and all $G \in \mathcal{C}$.

It turned out that nowhere dense classes have many equivalent and seemingly unrelated characterisations making it an extremely robust and natural concept [26].

Algorithmically, a characterisation of nowhere dense classes in terms of uniform quasi-wideness, a concept emerging from finite model theory [4], has proved to be extremely useful. A set $B \subseteq V(G)$ is $r$-independent in a graph $G$ if any two distinct vertices of $B$ have distance greater than $r$ in $G$.

Definition 2. A class $\mathcal{C}$ of graphs is uniformly quasi-wide if there are functions $N: \mathbb{N} \times \mathbb{N} \rightarrow \mathbb{N}$ and $s: \mathbb{N} \rightarrow \mathbb{N}$ such that for all $r, m \in \mathbb{N}$ and all subsets $A \subseteq V(G)$ for $G \in \mathcal{C}$ of size $|A| \geq N(r, m)$ there is a set $S \subseteq V(G)$ of size $|S| \leq s(r)$ and a set $B \subseteq A$ of size $|B| \geq m$ which is $r$-independent in $G-S$. The functions $N$ and $s$ are called the margin of the class $\mathcal{C}$. 
Theorem 3 (Nešetřil and Ossona de Mendez [24]). A class $\mathcal{C}$ of graphs is nowhere dense if, and only if, it is uniformly quasi-wide.

The first fixed-parameter algorithms for the DOMINATING SET problem on nowhere dense classes of graphs appeared in [5]. As observed in [5], uniform quasi-wideness can be made algorithmic in the sense that the sets $S$ and $B$ can be computed in polynomial time. This can be used to define bounded search tree algorithms for problems such as Dominating SET parameterized by the solution size $k$ as follows. As long as the set $A$ of non-dominated vertices is large enough we are guaranteed to find a 2 -independent subset $B$ of $A$ of size $k+1$ in $G$ once we removed a constant size set $S$ of vertices from $G$. As no two elements of $B$ can be dominated by a single element in $G-S$, it follows that the dominating set must contain an element of the constant size set $S$. Trying every subset of $S$ as a part of the dominating set and iterating this procedure until the number of non-dominated vertices is bounded by a function of the parameter yields a natural reduction. On the resulting structure one obtains the answer by brute force. With a little more effort this technique can be used to establish fixed-parameter algorithms for many other problems, see [5] for details.

A much more general result was achieved in [17]. Grohe et al. proved a very general algorithmic meta-theorem stating that first-order model-checking is fixed parameter tractable on nowhere dense classes of graphs (with the size of the formula as the parameter). This implies that a very broad and natural class of algorithmic problems is fixed-parameter tractable on nowhere dense classes of graphs. Again this proof uses uniform quasi-wideness in its construction.

More recently nowhere dense classes of graphs have also been studied in the context of kernelisation. In [9], it was shown that Dominating SeT and Distance- $r$ Dominating Set admit a linear kernel on bounded expansion classes and that Dominating SET admits an almost linear kernel on nowhere dense classes of graphs. A distance-r dominating set is a set $D \subseteq V(G)$ such that every vertex $u \in V(G)$ has distance at most $r$ to a vertex from $D$. However, the techniques used in [9] are not strong enough to show that also the Distance-r Dominating Set problem admits a polynomial kernel on nowhere dense classes of graphs. It was shown, however, that for every class $\mathcal{C}$ of graphs which is closed under taking subgraphs, if $\mathcal{C}$ admits a kernel for the DisTANCE$r$ Dominating SeT problem for every value of $r \in \mathbb{N}$, then $\mathcal{C}$ must be nowhere dense (under the assumption $\mathrm{W}[2] \neq \mathrm{FPT}$ ). These results were complemented by lower bounds for the closely related Connected Dominating Set problem, where we are looking for a dominating set $D$ which additionally must be connected. It was shown that there exists a class of bounded expansion which is closed under taking subgraphs that does not admit a polynomial kernel for CONNECTED Dominating SeT (unless NP $\subseteq$ coNP/poly).

Our contributions. From an algorithmic perspective, the main problem with the characterisation of nowhere dense classes by uniform quasi-wideness is that the functions $N$ and $s$ used in the definition of the class are established by iterated Ramsey arguments (see [24, 26]). Therefore, the function $N$ grows extremely fast and depends non-elementarily on the size of the excluded cliques in the definition of nowhere denseness. It follows that fixed-parameter algorithms using uniform quasiwideness, such as the algorithms in [5] mentioned above, have astronomical parameter dependence making them infeasible in practice even for very small parameter values.

Our first main result is to improve the bounds on uniform quasi-wideness dramatically. In fact, we can show that a class $\mathcal{C}$ is uniformly quasi-wide with margin $N: \mathbb{N} \times \mathbb{N} \rightarrow \mathbb{N}$ if, and only if, for every $r \in \mathbb{N}$ there is a polynomial $p_{r}(x)$ such that $\mathcal{C}$ is uniformly quasi-wide with margin $N^{\prime}(r, m) \leq p_{r}(m)$. This is a direct corollary of the following theorem, which we prove in Section 3 . 
Theorem 4. Let $\mathcal{C}$ be a nowhere dense class of graphs. For every $r \in \mathbb{N}$ there exists a polynomial $p_{r}(x)$ and a constant $s(r)$ such that for all $m \in \mathbb{N}$ the following holds. For all $G \in \mathcal{C}$ and all sets $A \subseteq V(G)$ of size at least $p_{r}(m)$, there is a set $S \subseteq V(G)$ of size at most $s(r)$ such that there is a set $B \subseteq A$ of size at least $m$ which is $r$-independent in $G-S$.

Furthermore, if $K_{c} \aleph_{r} G$ for all $G \in \mathcal{C}$, then $s(r) \leq c$ and there is an algorithm, that given an $n$-vertex graph $G \in \mathcal{C}, \varepsilon>0, r \in \mathbb{N}$ and $A \subseteq V(G)$ of size at least $p_{r}(m)$, computes a set $S$ of size at most $s(r)$ and an $r$-independent set $B \subseteq A$ in $G-S$ of size at least $m$ in time $\mathcal{O}\left(r \cdot c \cdot|A|^{c+1} \cdot n^{1+\varepsilon}\right)$.

Corollary 5. A class $\mathcal{C}$ of graphs is uniformly quasi-wide with margins $N: \mathbb{N} \times \mathbb{N} \rightarrow \mathbb{N}$ and $s: \mathbb{N} \rightarrow$ $\mathbb{N}$ if, and only if, it is uniformly quasi-wide with a polynomial margin $N^{\prime}(r, m) \leq p_{r}(m)$ and a margin $s^{\prime}(r) \leq c$ for a polynomial $p_{r}(x)$ and a constant $c$ depending on $r$ and $\mathcal{C}$ only.

Compare this result to a result of a similar flavour by Demaine and Hajiaghayi [6], stating that a minor closed class $\mathcal{C}$ of graphs has bounded local treewidth if, and only if, $\mathcal{C}$ has linearly bounded local treewidth.

The polynomial bounds on the margin of uniformly quasi-wide classes, and hence nowhere dense classes, give us a new tool to prove polynomial kernels. As our second main algorithmic result, proved in Section 4, we take a step towards solving an open problem stated in [9], to find an (almost) linear kernel for the Distance- $r$ Dominating Set problem on nowhere dense classes of graphs.

Theorem 6. For every fixed value $r \in \mathbb{N}$, there is a polynomial kernel for the Distance- $r$ Dominating Set problem on every nowhere dense class of graphs.

We remark that in [8] it was already shown that for classes $\mathcal{C}$ that are closed under taking subgraphs, if $\mathcal{C}$ admits a kernel for the Distance- $r$ Dominating Set problem for every $r \in \mathbb{N}$, then $\mathcal{C}$ must be nowhere dense (under the standard assumption that FPT $\neq \mathrm{W}[2]$ ). Hence, under this assumption, the theorem implies that a class $\mathcal{C}$ which is closed under taking subgraphs admits a kernel for the Distance- $r$ Dominating SeT problem for every $r \in \mathbb{N}$ if, and only if, it admits a polynomial kernel for every $r \in \mathbb{N}$.

As another consequence of Theorem 4 we can dramatically improve the parameter dependence of the Connected Dominating Set problem. See Section 5 for a proof of the following theorem.

Theorem 7. Let $\mathcal{C}$ be a nowhere dense class of graphs. Then there is a polynomial $p(x)$ and an algorithm running in time $2^{p(k)} \cdot n^{1+\varepsilon}$ which, given an n-vertex graph $G, \varepsilon>0$ and a number $k$ as input, decides whether $G$ contains a connected dominating set of size $k$.

We believe that Theorem 4 can allow to reduce the parameter dependence for further fixedparameter algorithms on nowhere dense classes such as the algorithms developed in [5] .

We prove our results using tools from a branch of model theory known as stability theory. Stability was introduced by Shelah as a notion of well-behaved first-order logic theories. In a recent paper, Malliaris and Shelah [20] used the model theoretic tools to study classes of stable graphs. Stable classes of graphs are much more general than nowhere dense classes of graphs, but the two concepts coincide on classes of graphs closed under taking subgraphs [1]. The focus of [20] is on proving very strong regularity lemmas for stable graphs. For obtaining these lemmas they prove a very nice technical lemma, Theorem 15 below, on the existence of long $\Delta$-indiscernible sequences in 
stable graphs, where $\Delta$ is a finite set of first-order formulas. We are going to use $\Delta$-indiscernible sequences to extract large $r$-independent sets for properly defined formula sets $\Delta$. See Section 2 for the definition of $\Delta$-indiscernible sequences. One of the technical tools we develop in this paper is to make this lemma of [20] algorithmic so that we can apply it in our algorithms.

We believe that stable classes will be very interesting for future algorithmic research and may be a good candidate for a generalisation of nowhere dense classes with good algorithmic properties towards classes of graphs which are no longer closed under taking subgraphs (but e.g. are only closed under taking induced subgraphs). Our technical results here may therefore be of independent interest as a first step towards understanding the algorithmic context of stable classes of graphs.

\section{$2 \quad$ Stability and Indiscernibles}

Graphs. We use standard graph theoretical notation and refer to [7] for reference. Let $G$ be an undirected graph. We write $N(v)$ for the set of neighbours of a vertex $v \in V(G)$ and $v$ itself and $N_{r}(v)$ for the set of vertices at distance at most $r$ from $v$, again including $v$. A set $W \subseteq V(G)$ is called $r$-independent in $G$, if all distinct $u, v \in W$ have distance greater than $r$ in $G$.

Ladder index, branching index and VC-dimension. Let $G$ be a directed graph. The ladder index of $G$ is the largest number $k$ such that there are $v_{1}, \ldots, v_{k}, w_{1}, \ldots, w_{k} \in V(G)$ with

$$
\left(v_{i}, w_{j}\right) \in E(G) \Leftrightarrow i \leq j .
$$

The figure shows a graph with ladder index 6 (imagine the edges to be directed from $v_{i}$ to $w_{j}$ ).

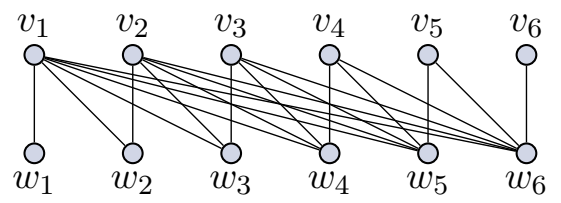

If $\tau$ is a word over an alphabet $\Sigma$ and $a \in \Sigma$, then $\tau \cdot a$ denotes the concatenation of $\tau$ and $a$. The branching index of $G$ is the largest number $\ell$ such that there are vertices $u_{\sigma_{1}}, \ldots, u_{\sigma_{2 \ell} \ell} \in V(G)$, indexed by the words over the alphabet $\{0,1\}$ of length exactly $\ell$, and vertices $v_{\tau_{1}}, \ldots, v_{\tau_{2} \ell_{-1}}$, indexed by the words over $\{0,1\}$ of length strictly smaller than $\ell$, such that if $\tau_{j} \cdot a$ is a (not necessarily proper) prefix of $\sigma_{i}$, then $\left(u_{\sigma_{i}}, v_{\tau_{j}}\right) \in E(G)$ if, and only if, $a=1$. The vertices $u_{\sigma_{1}}, \ldots, u_{\sigma_{2} \ell} \in V(G)$ are called the leaves of the tree, the vertices $v_{\tau_{1}}, \ldots, v_{\tau_{2} \ell_{-1}}$ are its inner nodes. Note that we describe edges only between inner nodes and leaves. Intuitively, a leaf $u$ is connected to its predecessors $v$ such that $u$ is a right successor of $v$ and not to its predecessors such that it is a left successor, while we make no assumptions on edges between different branches. We call the graph induced by vertices $u_{\sigma_{1}}, \ldots, u_{\sigma_{2} \ell}$ and $v_{\tau_{1}}, \ldots, v_{\tau_{2} \ell_{-1}}$ a branching witness for $G$ of index $\ell$.

The ladder index and branching index of $G$ are closely related, as shown by the next lemma.

Lemma 8 ([19], Lemma 6.7.9, p. 313). Let $G$ be a directed graph. If $G$ has branching index $k$, then $G$ has ladder index smaller than $2^{k+1}$. If $G$ has ladder index $k$, then $G$ has branching index smaller than $2^{k+2}-2$. 
We come to the definition of VC-dimension. Let $A$ be a set and let $\mathcal{F} \subseteq \mathcal{P}$ ow $(A)$ be a family of subsets of $A$. For a set $X \subseteq A$ let

$$
X \cap \mathcal{F}:=\{X \cap F: F \in \mathcal{F}\} .
$$

The set $X$ is shattered by $\mathcal{F}$ if

$$
X \cap \mathcal{F}=\operatorname{Pow}(X) .
$$

The Vapnik-Chervonenkis-dimension, short $V C$-dimension, of $\mathcal{F}$ is the maximum size of a set $X$ that is shattered by $\mathcal{F}$. Note that if $X$ is shattered by $\mathcal{F}$, then every subset of $X$ is shattered by $\mathcal{F}$.

The following theorem was first proved by Vapnik and Chervonenkis [33], and independently by Sauer [30] and Shelah [31]. It is often called the Sauer-Shelah-Lemma in the literature.

Theorem 9 (Sauer-Shelah-Lemma). If $|A| \leq m$ and $\mathcal{F} \subseteq \mathcal{P}$ ow $(A)$ has $V C$-dimension d, then

$$
|\mathcal{F}| \leq \sum_{i=0}^{d}\left(\begin{array}{c}
m \\
i
\end{array}\right) \leq m^{d}+1 .
$$

The VC-dimension of an undirected graph $G$ is the VC-dimension of the family of sets

$$
\mathcal{F}=\{N(v): v \in V(G)\}
$$

First-order logic. For extensive background on first-order logic, we refer the reader to [19]. For our purpose, it suffices to define first-order logic over the vocabulary of graphs (with constant symbols from a given parameter set).

Let $A$ be a set. We call $L(A):=\{E\} \cup A$ the vocabulary of graphs with parameters from $A$. First-order formulas over $L(A)$ are formed from atomic formulas $x=y$ and $E(x, y)$, where $x, y$ are variables (we assume that we have an infinite supply of variables) or elements of $A$ treated as constant symbols, by the usual Boolean connectives $\neg$ (negation), $\wedge$ (conjunction), and $\vee$ (disjunction) and existential and universal quantification $\exists x, \forall x$, respectively. The free variables of a formula are those not in the scope of a quantifier, and we write $\varphi\left(x_{1}, \ldots, x_{k}\right)$ to indicate that the free variables of the formula $\varphi$ are among $x_{1}, \ldots, x_{k}$. We often abbreviate a tuple $\left(x_{1}, \ldots, x_{k}\right)$ by $\bar{x}$ and let the context determine the length of a tuple $\bar{x}$.

To define the semantics, we inductively define a satisfaction relation $\models$. Let $G$ be a graph and $A \subseteq V(G)$. For an $L(A)$-formula $\varphi\left(x_{1}, \ldots, x_{k}\right)$, and $v_{1}, \ldots, v_{k} \in V(G), G \models \varphi\left(v_{1}, \ldots, v_{k}\right)$ means that $G$ satisfies $\varphi$ if the free variables $x_{1}, \ldots, x_{k}$ are interpreted by $v_{1}, \ldots, v_{k}$ and the parameters $a \in$ $A$ (formally treated as constant symbols) used in the formula are interpreted by the corresponding element of $A$ in $G$, respectively. If $\varphi\left(x_{1}, x_{2}\right)=E\left(x_{1}, x_{2}\right)$ is atomic, then $G \models \varphi\left(v_{1}, v_{2}\right)$ if $\left(v_{1}, v_{2}\right) \in$ $E(G)$. The meaning of the equality symbol, the Boolean connectives, and the quantifiers is as expected. For a formula $\varphi\left(x_{1}, \ldots, x_{k}, y_{1}, \ldots, y_{\ell}\right)$ and $v_{1}, \ldots, v_{\ell} \in V(G)$ (treated as a sequence of parameters), we write $\varphi\left(x_{1}, \ldots, x_{k}, v_{1}, \ldots, v_{\ell}\right)$ for the formula with free variables $x_{1}, \ldots, x_{k}$ where each occurrence of the variable $y_{i}$ in $\varphi$ is replaced by the constant symbol $v_{i}$.

Let $\Delta$ be a set of formulas, let $G$ be a graph and let $A \subseteq V(G)$. Let $v \in V(G)$. The $\Delta$-type of vertex $v$ in $G$ over the parameters $A$ is the set

$$
\begin{aligned}
\operatorname{tp}_{\Delta}(G, A, v): & =\left\{\varphi\left(x_{1}, v_{1}, \ldots, v_{k}\right): \varphi\left(x_{1}, y_{1}, \ldots, y_{k}\right) \in \Delta, v_{1}, \ldots, v_{k} \in A, G \models \varphi\left(v, v_{1}, \ldots, v_{k}\right)\right\} \\
& \cup\left\{\neg \varphi\left(x_{1}, v_{1}, \ldots, v_{k}\right): \varphi\left(x_{1}, y_{1}, \ldots, y_{k}\right) \in \Delta, v_{1}, \ldots, v_{k} \in A, G \not \models \varphi\left(v, v_{1}, \ldots, v_{k}\right)\right\} .
\end{aligned}
$$

The set of $\Delta$-types realised in $G$ over $A$ is the set $S_{\Delta}(G, A):=\left\{\operatorname{tp}_{\Delta}(G, A, v): v \in V(G)\right\}$. 
Example 10. Let $\Delta^{r}$ be the set consisting of the single formula $\varphi_{r}\left(x_{1}, y_{1}\right)$, stating that the elements $x_{1}$ and $y_{1}$ have distance at most $r$ in a graph $G$. Let $G$ be a graph and let $A \subseteq V(G)$.

1. For $v \in V(G)$, we can identify $\operatorname{tp}_{\Delta^{r}}(G, A, v)$ with $N_{r}(v) \cap A$, in the sense that $a \in N_{r}(v) \cap A$ if, and only if, the formula $\varphi_{r}\left(x_{1}, a\right) \in \operatorname{tp}_{\Delta^{r}}(G, A, v)$.

2. If $G$ is an arbitrary graph, then we can have $\left|S_{\Delta^{1}}(G, A)\right|=2^{|A|}$. If $G$ has VC-dimension $k$, then $\left|S_{\Delta^{1}}(G, A)\right| \leq|A|^{k}+1$ according to the Sauer-Shelah-Lemma. If $G$ comes from a nowhere dense class of graphs and $\varepsilon>0$, then we have $\left|S_{\Delta^{1}}(G, A)\right| \leq|A|^{1+\varepsilon}$ for all sufficiently large $A$. This follows from Lemma 4.11(2) of [16].

Using tools from stability theory, a much more general result can be established (Theorem 13 below).

First-order interpretations and stability. Let $\varphi\left(x_{1}, \ldots, x_{k}\right)$ with $k \geq 2$ be a first-order formula and let $G$ be a graph. For every ordered partition $\left(x_{i_{1}}, \ldots, x_{i_{\ell}}\right),\left(x_{i_{\ell+1}}, \ldots, x_{i_{k}}\right)$ of the variables $x_{1}, \ldots, x_{k}$ we define a directed graph $G_{\varphi\left(\left(x_{i_{1}}, \ldots, x_{i_{\ell}}\right),\left(x_{i_{\ell+1}}, \ldots, x_{i_{k}}\right)\right)}$ which has as its vertices the $\ell$-tuples $\left(v_{1}, \ldots, v_{\ell}\right)$ and $k-\ell$-tuples $\left(v_{\ell+1}, \ldots, v_{k}\right)$ of vertices of $G$ and all $\operatorname{arcs}\left(\left(v_{1}, \ldots, v_{\ell}\right),\left(v_{\ell+1}, \ldots, v_{k}\right)\right)$ such that $G \models \varphi\left(v_{i_{1}}, \ldots, v_{i_{k}}\right)$. If the variable partition is of relevance, we will always denote the formula as $\varphi\left(\left(x_{i_{1}}, \ldots, x_{i_{\ell}}\right),\left(x_{i_{\ell+1}}, \ldots, x_{i_{k}}\right)\right)$.

Example 11. Let $\varphi_{r}\left(x_{1}, x_{2}\right)$ be the formula from Example 10 (where we renamed the variables to match the above definition of interpretations). Then $G_{\varphi_{r}\left(x_{1}, x_{2}\right)}$ has the same vertex set as $G$ and any two vertices are joined by an edge in $G_{\varphi_{r}\left(x_{1}, x_{2}\right)}$ if, and only if, their distance is at most $r$ in $G$.

A formula $\varphi\left(\left(x_{i_{1}}, \ldots, x_{i_{\ell}}\right),\left(x_{i_{\ell+1}}, \ldots, x_{i_{k}}\right)\right)$ with an ordered partition of its free variables is stable on a class $\mathcal{C}$ of graphs, if there is an integer $s$ such that for every graph $G \in \mathcal{C}$ the graph $G_{\varphi\left(\left(x_{i_{1}}, \ldots, x_{i_{\ell}}\right),\left(x_{i_{\ell+1}}, \ldots, x_{i_{k}}\right)\right)}$ has ladder index at most $s$. A class $\mathcal{C}$ of graphs is stable if every formula with every partition of its free variables is stable on $\mathcal{C}$.

As proved by Adler and Adler in [1] (based on work of Podewski and Ziegler [28]), stable classes properly extend the concept of nowhere dense classes.

Theorem 12 ([1]). Every nowhere dense class of graphs $\mathcal{C}$ is stable, that is, for every formula $\varphi\left(\left(x_{i_{1}}, \ldots, x_{i_{\ell}}\right),\left(x_{i_{\ell+1}}, \ldots, x_{i_{k}}\right)\right)$ with every ordered partition of its free variables there is an integer $s$ such that for every graph $G \in \mathcal{C}$ the graph $G_{\varphi\left(\left(x_{i_{1}}, \ldots, x_{i_{\ell}}\right),\left(x_{i_{\ell+1}}, \ldots, x_{i_{k}}\right)\right)}$ has ladder index at most $s$.

Note that the converse is not true, as, e.g., the class of complete graphs is stable but not nowhere dense. In particular, stable classes are possibly not closed under taking subgraphs. We remark that in [20] a stable class of graphs is defined as a class $\mathcal{C}$ such that the ladder index of every graph $G \in \mathcal{C}$ is bounded by a constant $s$ depending only on $\mathcal{C}$ (that is, [20] does not demand closure under interpretations).

The following theorem is easily proved using the Sauer-Shelah Lemma.

Theorem 13 (see [32], Theorem II.4.10(4) and II.4.11(4), p.74). Let $\mathcal{C}$ be a stable class of graphs and let $\Delta$ be a finite set of first-order formulas. There exists an integer s such that for all $G \in \mathcal{C}$ and all $A \subseteq V(G)$ with $|A| \geq 2$ it holds that $\left|S_{\Delta}(G, A)\right| \leq|A|^{s}$. 
Corollary 14. Let $\mathcal{C}$ be a nowhere dense class of graphs and let $r$ be a positive integer. There is an integer s such that for all $G \in \mathcal{C}$ and all $A \subseteq V(G)$ it holds that

$$
\left|\left\{N_{r}(v) \cap A: v \in V(G)\right\}\right| \leq|A|^{s} .
$$

Indiscernible sequences. Let $G$ be a graph and let $\Delta$ be a set of formulas. A sequence $\left(v_{1}, \ldots, v_{\ell}\right)$ of vertices of $G$ is $\Delta$-indiscernible if for every formula $\varphi\left(x_{1}, \ldots, x_{k}\right) \in \Delta$ with $k$ free variables and any two increasing sequences $1 \leq i_{1}<\ldots<i_{k} \leq \ell, 1 \leq j_{1}<\ldots<j_{k} \leq \ell$ of integers, it holds that

$$
G \models \varphi\left(v_{i_{1}}, \ldots, v_{i_{k}}\right) \Leftrightarrow G \models \varphi\left(v_{j_{1}}, \ldots, v_{j_{k}}\right) .
$$

The following theorem forms the basis of our construction. The proof follows immediately from Theorem 3.5, Item (2) of [20] and parallels that proof, however, we provide a proof of the theorem because we will provide a precise analysis for the nowhere dense case in Section 3.

Theorem 15. Let $\mathcal{C}$ be a stable class of graphs and let $\Delta$ be a finite set of first-order formulas. There is a polynomial $p(x)$ such that for all $G \in \mathcal{C}$, every positive integer $m$ and every sequence $\left(v_{1}, \ldots, v_{\ell}\right)$ of vertices of $G$ of length $\ell=p(m)$, there exists a sub-sequence $\left(v_{i_{1}}, \ldots, v_{i_{m}}\right)$ of $\left(v_{1}, \ldots, v_{\ell}\right)$ of length $m$ which is $\Delta$-indiscernible, $1 \leq i_{1}<\ldots<i_{m} \leq \ell$.

Furthermore, there is an algorithm that given an $n$-vertex graph $G \in \mathcal{C}$ and a sequence $\left(v_{1}, \ldots, v_{\ell}\right) \subseteq$ $V(G)$, computes a $\Delta$-indiscernible sub-sequence of $\left(v_{1}, \ldots, v_{\ell}\right)$ of length at least $m$. The running time of the algorithm is in $\mathcal{O}\left(|\Delta| \cdot k \cdot \ell^{k+1} \cdot n^{q} \cdot a(n) \cdot \lambda(\Delta)\right)$, where $k$ is the maximal number of free variable, $q$ is the maximal quantifier-rank of a formula of $\Delta, a(n)$ is the time required to test adjacency between two vertices and $\lambda(\Delta)$ is the length of a longest formula in $\Delta$.

Proof. Let $G \in \mathcal{C}$ and let $\left(v_{1}, \ldots, v_{\ell}\right)$ be a sequence of vertices of $G$. We prove that for every formula $\varphi\left(x_{1}, \ldots, x_{k}\right) \in \Delta$ there is a $\{\varphi\}$-indiscernible subsequence of length at least $f^{k}(\ell)$, where $f(\ell)=\left(\frac{\ell}{t}\right)^{\frac{1}{t r+t+1}}-k$ for constants $r, t$ depending only on $\varphi$ and $\mathcal{C}$. The claim of the theorem then follows by iteratively extracting $\{\varphi\}$-indiscernible sequences for all $\varphi \in \Delta$ and combining the polynomials accordingly.

Let $\varphi\left(x_{1}, \ldots, x_{k}\right) \in \Delta$. Let $G \in \mathcal{C}$ and let $\left(v_{1}, \ldots, v_{\ell}\right)$ be a sequence of vertices of $G$. Let $A:=\left\{v_{1}, \ldots, v_{\ell}\right\}$. As $\mathcal{C}$ is stable, according to Theorem 13, there is an integer $r$ such that $\left|S_{\{\varphi\}}(G, A)\right| \leq|A|^{r}$. Here, we consider the sets $S_{\left\{\varphi\left(\left(x_{i}\right),\left(x_{1}, \ldots, x_{i-1}, x_{i+1}, \ldots, x_{k}\right)\right)\right\}}(G, A)$ for every partition $\varphi\left(\left(x_{i}\right),\left(x_{1}, \ldots, x_{i-1}, x_{i+1}, \ldots, x_{k}\right)\right)$ of the free variables of $\varphi$ and we choose $r$ such that it works for every such partition of the variables.

We prove by induction on $0 \leq m \leq k$ that there exists a sub-sequence $\bar{u}_{m}$ of $\left(v_{1}, \ldots, v_{\ell}\right)$ with

1. $\left|\bar{u}_{m}\right| \geq f^{m}(\ell)$ and

2. for all sub-sequences $\bar{a}=a_{1}, \ldots, a_{k}$ and $\bar{b}=b_{1}, \ldots, b_{k}$ of $\bar{u}_{m}$, if $a_{i}=b_{i}$ for all $i \in\{1, \ldots, k-m\}$, then

$$
G \models \varphi(\bar{a}) \Leftrightarrow G \models \varphi(\bar{b}) .
$$


Note that the elements of a sub-sequence of a sequence preserve their respective order.

For $m=0$ there is nothing to show, we can take $\bar{u}_{0}=\left(v_{1}, \ldots, v_{\ell}\right)$ of length $f^{0}(\ell)=\ell$.

For $m+1$ assume that $\bar{u}_{m}=\left(v_{1}, \ldots, v_{\ell(m)}\right)$ is constructed as required. We define

$$
\varphi^{m}=\varphi\left(\left(x_{k-m}\right),\left(x_{1}, \ldots, x_{k-m-1}, v_{\ell(m)-m+1}, \ldots, v_{\ell(m)}\right)\right),
$$

that is, we fix the interpretation of the last $m$ free variables as the last $m$ elements of the sequence $\bar{u}_{m}$.

We construct a tree $T$ whose vertices are elements from $\bar{u}_{m}$, except for the root, which we label $\emptyset$. We attach $v_{1}$ as a child of the root $\emptyset$. In the following, by a maximal element $z$ in the tree $T$ satisfying some condition we always mean an element of $T$ satisfying this condition which is as far away from the root as possible in the sense that in the subtree of $T$ rooted at $z$ no other element satisfies this condition.

By induction, assume that for some $j<\ell(m)$ all $v_{i}$ with $i<j$ are inserted in the tree and we want to insert $v_{j}$. For a vertex $v$ of the tree let $P(v)$ be the path from the root to $v$ excluding $v$ and for a child $v$ of the root let $P(v)$ be the empty path. Let $w$ be a maximal vertex that realises the same $\left\{\varphi^{m}\right\}$-type over $P(w)$ as $v_{j}$ (that is, no successor $z$ of $w$ realises the same $\left\{\varphi^{m}\right\}$-type over $P(z)$ as $v_{j}$ ), or $w=\emptyset$ if no such vertex exists. We insert $v_{j}$ as a new child of $w$.

We call the resulting tree $T$ a type tree. For two vertices $v$ and $w$ we write $v<_{T} w$ if $v \in P(w)$. The tree constructed in this way satisfies three properties:

- if $v=v_{i}<_{T} w=v_{j}$, then $i<j$, that is, paths in the type tree respect the order of $\bar{u}_{m}$,

- if $v<_{T} w$, then $v$ and $w$ have the same $\left\{\varphi^{m}\right\}$-types over $P(v)$, and

- if $v \neq w$ and neither $v<_{T} w$ nor $w<_{T} v$, then $v$ and $w$ realise different $\left\{\varphi^{m}\right\}$-types over $P(v) \cap P(w)$ and they realise the same $\left\{\varphi^{m}\right\}$-type over $P(z)$, where $z$ is the maximal element of $P(v) \cap P(w)$.

We show that there exists a sufficiently long branch of $T$ that can be used as $\bar{u}_{m+1}$. Let $\bar{a}=a_{1}, \ldots, a_{k}$ and $\bar{b}=b_{1}, \ldots, b_{k}$ be sub-sequences of $\bar{u}_{m}$ such that, first, $a_{i}=b_{i}$ for all $i \in$ $\{1, \ldots, k-m-1\}$, and, second, both $\bar{a}$ and $\bar{b}$ are subsequences of a path $P(w)$ in $T$ for some $w$. If $a_{k-m}=b_{k-m}$, Condition 2 is proven for $\bar{u}_{m+1}$, so we can assume without loss of generality that $a_{k-m}<_{T} b_{k-m}$. For all sequences $\bar{d}_{1}$ and $\bar{d}_{2}$ we abbreviate $G \models \varphi\left(\bar{d}_{1}\right) \Leftrightarrow G \models \varphi\left(\bar{d}_{2}\right)$ by $\varphi\left(\bar{d}_{1}\right) \equiv_{G} \varphi\left(\bar{d}_{2}\right)$. Then

$$
\varphi\left(a_{1}, \ldots, a_{k-m-1}, a_{k-m}, \ldots, a_{k}\right) \equiv_{G} \varphi\left(a_{1}, \ldots, a_{k-m-1}, a_{k-m}, v_{\ell(m)-m+1}, \ldots, v_{\ell(m)}\right)
$$

by the induction hypothesis, as the arguments coincide on the first $k-m$ arguments. As $a_{k-m}$ and $b_{k-m}$ realise the same $\varphi^{m}$-type over $P\left(a_{k-m}\right)$, we obtain

$$
\varphi\left(a_{1}, \ldots, a_{k-m-1}, a_{k-m}, v_{\ell(m)-m+1}, \ldots, v_{\ell(m)}\right) \equiv_{G} \varphi\left(a_{1}, \ldots, a_{k-m-1}, b_{k-m}, v_{\ell(m)-m+1}, \ldots, v_{\ell(m)}\right)
$$

and, using the induction hypothesis again and the condition that $a_{i}=b_{i}$ for $i \leq k-m-1$,

$$
\varphi\left(a_{1}, \ldots, a_{k-m-1}, b_{k-m}, v_{\ell(m)-m+1}, \ldots, v_{\ell(m)}\right) \equiv_{G} \varphi\left(a_{1}, \ldots, a_{k-m-1}, b_{k-m}, b_{k-m+1}, \ldots, b_{k}\right) .
$$

This proves Condition 2. 
It remains to show that the tree has a sufficiently long branch. For this, we want to show that $T$ does not contain a large complete binary subtree. Consider the graph

$$
G_{B}=G_{\varphi\left(\left(x_{k-m}\right),\left(x_{1}, \ldots, x_{k-m-1}, v_{\ell(m)-m+1}, \ldots, v_{\ell(m)}\right)\right)}
$$

consisting of vertices represented by single vertices of $G$ (first type) and those represented by $k-1$ tuples (second type). As by assumption $\mathcal{C}$ is stable, according to Theorem 12 , the graph $G_{B}$ as an interpretation of $G$ has ladder index at most $s$ for some constant $s$, and hence according to Lemma 8, its branching index is bounded by $t:=2^{s+2}-2$. Let $S$ be a complete binary subtree of $T$. We show that we can construct a branching witness for $G_{B}$ with the same leaves as $S$ (note that the vertices of first type are actual vertices of $G$ ). This implies that $S$ can have depth at most $t$.

By construction of $S$, all distinct leaves $v, w$ of $S$ have a different $\left\{\varphi^{m}\right\}$-type over $P(v) \cap P(w)$ while they have the same type over $P(z)$, where $z$ is the maximal element of $P(v) \cap P(w)$. In particular, there is a tuple $\left(a_{1}, \ldots, a_{k-m-1}\right) \in P(z)$ such that $G \models \varphi\left(a_{1}, \ldots, a_{k-m-1}, u, v_{\ell(m)-m+1}, \ldots, v_{\ell(m)}\right)$ for all right successors $v$ of $z$, and such that $G \not \models \varphi\left(a_{1}, \ldots, a_{k-m-1}, w, v_{\ell(m)-m+1}, \ldots, v_{\ell(m)}\right)$ for all left successors $w$ of $z$. Observe that all inner vertices of the so constructed branching witness are distinct as no other tuple contains the element $a_{k-m-1}$ at position $k-m$. Hence we constructed a branching witness of index $t$ as claimed.

We now assign to every vertex $v$ in the tree $T$ its binary rank, that is, the maximal height of a full binary tree that is a sub-graph of the sub-tree rooted at $v$ (compare to the stability rank of sets of formulas in [32]). The depth of a vertex $v$ in $T$ is $|P(v)|$.

Let $N_{\ell}^{s}$ be the set of vertices of the type tree $T$ with rank $s$ and depth $\ell$. For $\ell>0$ let $X_{\ell}^{s} \subseteq N_{\ell}^{s}$ be the set vertices from $N_{\ell}^{s}$ whose direct predecessor has rank $s$ and let $Y_{\ell}^{s} \subseteq N_{\ell}^{s}$ be the set vertices from $N_{\ell}^{s}$ whose direct predecessors have rank $s+1$ (we may assume that we have maximal branching, hence all predecessors have rank $s$ or $s+1)$.

Then $N_{\ell}^{s} \subseteq X_{\ell}^{s} \cup Y_{\ell}^{s}$. Define

$$
n_{\ell}^{s}=\left|N_{\ell}^{s}\right|, \quad x_{\ell}^{s}=\left|X_{\ell}^{s}\right| \quad \text { and } \quad y_{\ell}^{s}=\left|Y_{\ell}^{s}\right| .
$$

A vertex $v$ of depth $d$ can have at most $(d+m+1)^{r}$ direct successors (recall that for every set $A$ we have $\left.\left|S_{\{\varphi\}}(G, A)\right| \leq|A|^{r}\right)$. This is because every two such successors have different $\varphi^{m}$-types over $P(v) \cup\{v\}$, so there are $|P(v)|+1$ predecessors and, additionally, $m$ parameters that are fixed in the formula $\varphi^{m}$.

The following inequalities hold:

1. $x_{\ell+1}^{s} \leq n_{\ell}^{s}$, because the direct predecessor $w$ of any vertex in $X_{\ell}^{s}$ has at most one direct successor of rank $s$ (otherwise $w$ had rank $s+1$ ),

2. $y_{\ell+1}^{s} \leq n_{\ell}^{s+1} \cdot(\ell+m+1)^{r}$,

3. $n_{\ell+1}^{s} \leq n_{\ell}^{s}+n_{\ell}^{s+1} \cdot(\ell+m+1)^{r}$,

4. $n_{0}^{s}=0$ for $1 \leq s<t$,

5. $n_{\ell}^{t} \leq 1$ where $t$ is the rank of the root.

Claim 1. $n_{\ell+1}^{t-s} \leq(\ell+m+1)^{s(r+1)}$. 
Proof. For $s=1$ we show the statement by induction on $\ell$. For $\ell=0$ first (3) and then (4) and (5) give us

$$
n_{1}^{t-1} \leq n_{0}^{t-1}+n_{0}^{t}(\ell+m+1)^{r} \leq(\ell+m+1)^{r} .
$$

For $\ell+1$ using first (3) and then the induction hypothesis (for $\ell$ ) and (5) we obtain

$$
n_{\ell+1}^{t-1} \leq n_{\ell}^{t-1}+n_{\ell}^{t}(\ell+m+1)^{r} \leq(\ell+m)^{r+1}+(\ell+m+1)^{r} \leq(\ell+m+1)^{r+1} .
$$

For $s+1$ the proof is again by induction on $\ell$. For $\ell=0$,

$$
n_{1}^{t-(s+1)} \leq n_{0}^{t-(s+1)}+n_{0}^{t-s}(\ell+m+1)^{r} \leq(\ell+m+1)^{r}
$$

and for $s+1$ as above and using the induction hypothesis for $s$,

$$
\begin{aligned}
n_{\ell+1}^{t-(s+1)} \leq n_{\ell}^{t-(s+1)}+n_{\ell}^{t-s}(\ell+m+1)^{r} & \leq(\ell+m)^{(s+1)(r+1)}+(\ell+m)^{s(r+1)}(\ell+m+1)^{r} \\
& \leq(\ell+m+1)^{(s+1)(r+1)} .
\end{aligned}
$$

The total number $n_{\ell+1}$ of vertices of depth $\ell+1$ is then

$$
n_{\ell+1} \leq \sum_{s \leq t} n_{\ell+1}^{t-s} \leq \sum_{s \leq t}(\ell+m+1)^{s(r+1)} \leq t(\ell+m+1)^{t(r+1)} .
$$

Now the number of vertices in a tree of depth at most $d$ (including the root at depth 0 ) is

$$
N=1+\sum_{\ell<d} n_{\ell+1} \leq 1+\sum_{\ell<d} t(\ell+m+1)^{t(r+1)}<t(d+m+1)^{t(r+1)+1}
$$

and thus $d>\left(\frac{N}{t}\right)^{\frac{1}{t r+t+1}}-m-1$, so if $\left|\bar{u}_{m}\right|>t(d+m+1)^{t(r+1)+1}$, then there is a branch of length at least $\left(\frac{N}{t}\right)^{t r+t+1}-m$. Replacing $m$ with $k$ we obtain a slightly worse bound that, however, does not depend on the induction step.

In $k$ steps we extract a sequence $\bar{u}$ of length at least $f^{(k)}(\ell(m))$, where $f(\ell)=\left(\frac{\ell}{t}\right)^{\frac{1}{t r+t+1}}-k$.

It remains to analyse the running time of the constructive procedure described above. It suffices to show that the sequence constructed in the inductive step of the above proof can be computed in polynomial time. First, the type tree $T$ is computed. We construct $T$ inductively as in the proof. While adding new vertices to the tree, we keep track of their height in the tree and we keep track of the longest branch. Thus after computing $T$, we just output the longest branch as $\bar{u}_{m+1}$.

For every vertex $v \in \bar{u}_{i}$, we search through the type tree to find the maximal element with the same $\left\{\varphi^{m}\right\}$-type to decide where to insert $v$. Here, we have to compare $v$ to less than $\ell$ elements.

To check the $\left\{\varphi^{m}\right\}$-type we perform a standard model-checking algorithm to verify whether $G \models \varphi(\bar{a}, v, \bar{v})$. Here $\bar{a}$ is an $m$-tuple of vertices on the path from the root to the current leaf (of length less than $\ell$ ) and $\bar{v}$ are the parameters from $\varphi^{m}$. Note that $m \leq k$ and the parameters in the formulas of $\varphi^{m}$ are fixed for each $m$. Hence, the check whether $G \models \varphi(\bar{a}, v, \bar{v})$ can be carried out in time $\mathcal{O}\left(n^{q} \cdot \lambda(\Delta) \cdot a(n)\right)$ and it takes time at most $\mathcal{O}\left(\ell^{k}\right)$ to iterate through all $m$-tuples $\bar{a}$.

Summing up, we need $\mathcal{O}\left(|\Delta| \cdot k \cdot \ell \cdot \ell^{k} \cdot n^{q} \cdot \lambda(\Delta) \cdot a(n)\right)=\mathcal{O}\left(|\Delta| \cdot k \cdot \ell^{k+1} \cdot n^{q} \cdot \lambda(\Delta) \cdot a(n)\right)$ steps to compute the final $\Delta$-indiscernible sequence. 
As a consequence we obtain the following corollary, which in particular applies to nowhere dense classes of graphs.

Corollary 16. Let $\mathcal{C}$ be a stable class of graphs and let $\Delta$ be an arbitrary finite set of formulas. Then there is a polynomial $p(x)$ such that for every positive integer $m$ and every sequence $\left(v_{1}, \ldots, v_{\ell}\right)$ of vertices of $G \in \mathcal{C}$ of length $\ell=p(m)$, there is a subsequence of length at least $m$ which is $\Delta$ indiscernible.

\section{Improved Bounds on Uniform Quasi-Wideness}

We can now show our first main theorem (Theorem 4), stating that a class is uniformly quasi-wide with margin $N: \mathbb{N} \times \mathbb{N} \rightarrow \mathbb{N}$ if, and only if, for every $r \in \mathbb{N}$ there is a polynomial margin $N^{\prime}(r, m)$. For convenience, we repeat the statement of the theorem here.

Theorem 4. Let $\mathcal{C}$ be a nowhere dense class of graphs. For every $r \in \mathbb{N}$ there exists a polynomial $p_{r}(x)$ and a constant $s(r)$ such that for all $m \in \mathbb{N}$ the following holds. For all $G \in \mathcal{C}$ and all sets $A \subseteq V(G)$ of size at least $p_{r}(m)$, there is a set $S \subseteq V(G)$ of size at most $s(r)$ such that there is a set $B \subseteq A$ of size at least $m$ which is $r$-independent in $G-S$.

Furthermore, if $K_{c} \aleph_{r} G$ for all $G \in \mathcal{C}$, then $s(r) \leq c$ and there is an algorithm, that given an $n$-vertex graph $G \in \mathcal{C}, \varepsilon>0, r \in \mathbb{N}$ and $A \subseteq V(G)$ of size at least $p_{r}(m)$, computes a set $S$ of size at most $s(r)$ and an $r$-independent set $B \subseteq A$ in $G-S$ of size at least $m$ in time $\mathcal{O}\left(r \cdot c \cdot|A|^{c+1} \cdot n^{1+\varepsilon}\right)$.

The proof of the theorem is based on the extraction of a large $\Delta$-indiscernible sequence from the set $A$ for a certain set $\Delta$ of formulas. Let $k \in \mathbb{N}$ and for $1 \leq i \leq k$ let

$$
\varphi_{i}\left(x_{1}, \ldots, x_{k}\right):=\exists y\left(\bigwedge_{1 \leq j \leq i} E\left(y, x_{j}\right) \wedge \bigwedge_{i<j \leq k} \neg E\left(y, x_{j}\right)\right)
$$

and let

$$
\psi_{i}\left(x_{1}, \ldots, x_{k}\right):=\exists y\left(\bigwedge_{i<j \leq k} E\left(y, x_{i}\right) \wedge \bigwedge_{1 \leq j \leq i} \neg E\left(y, x_{j}\right)\right) .
$$

The formula $\varphi_{i}\left(x_{1}, \ldots, x_{k}\right)$ states that there exists a vertex $v$ which is adjacent exactly to the first $i$ elements $x_{1}, \ldots, x_{i}$, while $\psi_{i}\left(x_{1}, \ldots, x_{k}\right)$ states that there exists a vertex which is non-adjacent exactly to the first $i$ elements $x_{1}, \ldots, x_{i}$. Let

$$
\Delta_{k}:=\left\{E\left(x_{1}, x_{2}\right)\right\} \cup\left\{\varphi_{1}, \ldots, \varphi_{k}, \psi_{1}, \ldots, \psi_{k}\right\} .
$$

Note that the formula $E\left(x_{1}, x_{2}\right)$ in $\Delta_{k}$ guarantees that the vertices of every $\Delta_{k}$-indiscernible sequence of vertices of a graph $G$ either form an independent set or a clique in $G$.

The crucial property we are going to use is stated as Claim 3.2 in [20]. Recall the definition of the ladder-index from Section 2.

Lemma 17 (Claim 3.2 of [20]). Let $k \in \mathbb{N}$ and let $G$ be a graph with ladder-index less than $k$. If $n \geq 4 k$ and $\left(v_{1}, \ldots, v_{n}\right)$ is a $\Delta_{k}$-indiscernible sequence in $G$ and $w \in V(G)$, then either

$$
\left|N(w) \cap\left\{v_{1}, \ldots, v_{n}\right\}\right|<2 k \text { or }\left|\left\{v_{1}, \ldots, v_{n}\right\} \backslash N(w)\right|<2 k .
$$


For nowhere dense classes we can derive even stronger properties of $\Delta_{k}$-indiscernible sequences. We need one more lemma, which follows easily from Lemma 4.11(2) of [16].

Lemma 18 (see Lemma 4.11(2) of [16]). Let $\mathcal{C}$ be a nowhere dense class of graphs. For every $\varepsilon>0$ there is an integer $n_{0}$ such that if $A \subseteq V(G)$ for $G \in \mathcal{C}$ with $|A| \geq n_{0}$, then

$$
|\{N(v) \cap A: v \in V(G)\}| \leq|A|^{1+\varepsilon} .
$$

Note that if $K_{k} \aleph_{1} G$, then $G$ does not contain a $k$-ladder and excludes the complete bipartite graph $K_{k, k}$ as a subgraph.

Lemma 19. Let $G$ be a graph with $K_{k} \aleph_{1} G$. There exists an integer $n_{1}=n_{1}(k)$ such that if $\left(v_{1}, \ldots v_{n}\right)$ is a $\Delta_{k}$-indiscernible sequence of length $n \geq n_{1}$, then every vertex $v \in V(G)$ is either connected to at most one vertex of $\left\{v_{1}, \ldots, v_{n}\right\}$ or to all of them.

Proof. As $K_{k} \npreceq_{1} G$, in particular, $G$ has ladder-index less than $k$ and excludes $K_{k, k}$ as a subgraph. If $n \geq 4 k$, according to Lemma 17, every vertex $w \in V(G)$ satisfies $\left|N(w) \cap\left\{v_{1}, \ldots, v_{n}\right\}\right|<2 k$ or $\left|\left\{v_{1}, \ldots, v_{n}\right\} \backslash N(w)\right|<2 k$. We first show that there are only a few vertices $w$ which satisfy $\left|\left\{v_{1}, \ldots, v_{n}\right\} \backslash N(w)\right|<2 k$. We will refer to these vertices as high degree vertices in the rest of the proof.

Assume there are at least $k$ high degree vertices. Fix a set $A$ of exactly $k$ of these vertices. Take as $B$ the set $\left(\bigcap_{w \in A} N(w)\right) \cap\left\{v_{1}, \ldots, v_{n}\right\}$. This set has order at least $n-2 k^{2}$. By definition of $\Delta_{k}$-indiscernibility, the vertices $v_{1}, \ldots, v_{n}$ either form an independent set or a clique in $G$. If $n \geq k$, they form an independent set by assumption. Hence $A \cap B=\emptyset$ and if $n \geq 2 k^{2}+k$, we find a complete bipartite graph $K_{k, k}$ as a subgraph of $G$, contradicting our assumption.

Assume towards a contradiction that there is a vertex $v \in V(G)$ which is connected to exactly $s$ vertices among $\left\{v_{1}, \ldots, v_{n}\right\}, 2 \leq s \leq n-1$. Then for some $i, 2 \leq i \leq k-1$, there is an increasing tuple $\left(v_{i_{1}}, \ldots, v_{i_{k}}\right)$ for $1 \leq i_{1}<\ldots<i_{k} \leq n$ such that $G \models \varphi_{i}\left(v_{i_{1}}, \ldots, v_{i_{k}}\right)$ or $G \models \psi_{i}\left(v_{i_{1}}, \ldots, v_{i_{k}}\right)$ : if $s \leq 2 k$, we can choose $i=2$, pick 2 neighbours of $v$ among $v_{1}, \ldots, v_{n}$ and $k-2$ non-neighbours which are either all smaller or all larger than the two neighbours and define $\left(v_{i_{1}}, \ldots, v_{i_{k}}\right)$ accordingly. Similarly, if $s>n-2 k$ we can choose $i=k-1$ and pick one non-neighbour of $v$ and $k-1$ neighbours to define $\left(v_{i_{1}}, \ldots, v_{i_{k}}\right)$.

Consider first the case that there is a tuple $\left(v_{i_{1}}, \ldots, v_{i_{k}}\right)$ such that $G \models \varphi_{2}\left(v_{i_{1}}, \ldots, v_{i_{k}}\right)$ (the case that $G \models \psi_{2}\left(v_{i_{1}}, \ldots, v_{i_{k}}\right)$ is completely analogous). By definition of $\Delta_{k}$-indiscernibility, every increasing $k$-tuple $\left(v_{j_{1}}, \ldots, v_{j_{k}}\right)$ satisfies $\varphi_{2}$, that is, there is an element $w \in V(G)$ such that $w$ is adjacent to $v_{j_{1}}$ and $v_{j_{2}}$ and not to $v_{j_{3}}, \ldots, v_{j_{k}}$. There are $\left(\begin{array}{l}n \\ k\end{array}\right)$ increasing $k$-tuples of elements of $\left(v_{1}, \ldots, v_{n}\right)$.

Every vertex $z$ with $a:=\left|\left\{v_{1}, \ldots, v_{n}\right\} \backslash N(z)\right|<2 k$ can take the role of this vertex $w$ for at most $\left(\begin{array}{c}n-a \\ 2\end{array}\right) \cdot\left(\begin{array}{c}a \\ k-2\end{array}\right)$ tuples: choose 2 vertices from the $n-a$ neighbours of $w$ and $k-2$ vertices from its $a$ non-neighbours among $\left\{v_{1}, \ldots, v_{n}\right\} \backslash N(z)$. We have at most $k$ of these high degree vertices as shown above. Hence at most $k \cdot\left(\begin{array}{c}n-1 \\ 2\end{array}\right) \cdot\left(\begin{array}{c}2 k-1 \\ k-2\end{array}\right) \in \mathcal{O}\left(n^{2}\right)$ tuples satisfy $\varphi_{2}$ because of high degree vertices.

Every vertex $z$ with $a:=\left|\left\{v_{1}, \ldots, v_{n}\right\} \cap N(z)\right|<2 k$ can play the role of $w$ for at most $\left(\begin{array}{l}a \\ 2\end{array}\right) \cdot\left(\begin{array}{l}n-a \\ k-2\end{array}\right) \leq$ $\left(\begin{array}{c}2 k-1 \\ 2\end{array}\right)\left(\begin{array}{c}n-2 \\ k-2\end{array}\right) \in \mathcal{O}\left(n^{k-2}\right)$ tuples. Denote by $x$ the number of small degree vertices.

Then it must hold that

$$
k \cdot\left(\begin{array}{c}
n-1 \\
2
\end{array}\right) \cdot\left(\begin{array}{c}
2 k-1 \\
k-2
\end{array}\right)+x \cdot\left(\begin{array}{c}
2 k-1 \\
2
\end{array}\right)\left(\begin{array}{l}
n-2 \\
k-2
\end{array}\right) \geq\left(\begin{array}{l}
n \\
k
\end{array}\right)
$$


from which we conclude that $x \geq c \cdot n^{2}$ for some fixed constant $0<c<1$ and all $n>n_{1}$ for sufficiently large $n_{1}$ (choose $\varepsilon=1 / 2$ and $n_{1}$ large enough such that we can apply Lemma 18). Without loss of generality we may assume that all small degree vertices induce distinct neighbourhoods on $A$ (realising one neighbourhood twice does not help to realise more types). Now, for sufficiently large $n$ we have a contradiction to Lemma 18 .

The proof for the case that there is a tuple $\left(v_{i_{1}}, \ldots, v_{i_{k}}\right)$ such that $G \models \varphi_{k-1}\left(v_{i_{1}}, \ldots, v_{i_{k}}\right)$ or $G \models \psi_{k-1}\left(v_{i_{1}}, \ldots, v_{i_{k}}\right)$ is similar. Here, the high degree vertices can cover at most $k \cdot\left(\begin{array}{l}n-1 \\ k-1\end{array}\right) \cdot(2 k-1) \in$ $\mathcal{O}\left(n^{k-1}\right)$ many tuples, while every low degree vertex can cover at most $\left(\begin{array}{c}2 k-1 \\ k-1\end{array}\right) \cdot(n-k-1) \in \mathcal{O}(n)$ many tuples.

We are ready to prove the main theorem.

Proof (Proof of Theorem 4). Let $\mathcal{C}$ be a nowhere dense class of graphs such that $K_{f(i)} \npreceq_{i} G$ for all $i$ and all $G \in \mathcal{C}$. Let $G \in \mathcal{C}$ and let $A \subseteq V(G)$. According to Corollary 16, we can extract from $A$ a large $\Delta_{f(1)}$-indiscernible sequence $B_{1}$. This requires $A$ to be only polynomially larger than $B_{1}$ according to the corollary.

We construct a sequence of graphs $G_{1}=G, G_{2}, \ldots$ and sequences $B_{1} \supseteq B_{2} \supseteq \ldots, \tilde{B}_{1} \supseteq \tilde{B}_{2} \supseteq \ldots$ and $S_{1}, S_{2}, \ldots$ of vertices of $G_{i}$ where $B_{i} \subseteq V(G), \tilde{B}_{i} \subseteq V\left(G_{i}\right)$ and $S_{i} \subseteq V(G) \cap V\left(G_{i}\right)$ such that for all $i$

1. $B_{i}$ is $2 i$-independent in $G-Z_{i}$ where $Z_{i}:=\bigcup_{j=1}^{i} S_{j}$,

2. $\left|S_{i}\right|<f(i)$,

3. $G_{i+1} \preccurlyeq_{i} G$ and

4. $\tilde{B}_{i}=\left\{G\left[N_{i}^{G-Z_{i}}(v)\right]: v \in B_{i}\right\}$ (recall that the vertices of $G_{i}$ as a minor of $G$ are subgraphs of $G)$.

As $K_{f(1)} \aleph_{1} G_{1}$, we can apply Lemma 19 and conclude that if $B_{1}$ is sufficiently large, then every vertex $v \in V\left(G_{1}\right)$ is either connected to at most one vertex of $B_{1}$ or to all vertices of $B_{1}$. Just as in the proof of Lemma 19, we show that there are less than $f(1)$ vertices that are adjacent to all vertices of $B_{1}$. We define $S_{1}$ as the set of all those (less than $f(1)$ ) high degree vertices: $S_{1}=\left\{v \in V(G): N\left(B_{1}\right) \subseteq N(v)\right\}$.

Note that $B_{1} \cap S_{1}=\emptyset$ if $\left|B_{1}\right| \geq f(1)$, as in this case, as above, the vertices of $B_{1}$ form an independent set. Hence every vertex $v \in V\left(G_{1}-S_{1}\right)$ is connected to at most one vertex of $B_{1}$, in other words, $B_{1}$ is a 2 -independent set in $G_{1}-S_{1}$. We hence established all of the above properties for $i=1$.

Let the sequences $G_{1}, G_{2}, \ldots, G_{i}, B_{1}, B_{2}, \ldots, B_{i}$ and $S_{1}, S_{2}, \ldots, S_{i}$ be defined for some fixed $i$. By Assumption $1, B_{i}$ is $2 i$-independent in $G-Z_{i}$, hence we can contract the disjoint $i$-neighbourhoods of the vertices of $B_{i}$. Let $\tilde{B}_{i}=\left\{G\left[N_{i}^{G-Z_{i}}(v)\right]: v \in B_{i}\right\}$ and let $H$ be the graph resulting from the contraction (i.e. $\tilde{B}_{i} \subseteq V(H)$ ). Let $\tilde{B}_{i}^{\prime} \subseteq \tilde{B}_{i}$ be a large independent set in $H$. We can obtain it by finding a large $\left\{E\left(x_{1}, x_{2}\right)\right\}$-indiscernible subsequence of $\tilde{B}_{i}$ in $H$ (by Theorem 15). Let $B_{i}^{\prime} \subseteq B_{i}$ be the set of vertices of $G$ such that $\tilde{B}_{i}^{\prime}=\left\{G\left[N_{i}^{G-Z_{i}}(v)\right]: v \in B_{i}^{\prime}\right\}$, i.e. the vertices of $B_{i}^{\prime}$ are the centres of $\tilde{B}_{i}^{\prime}$. Define the graph $G_{i}^{\prime}$ to be the depth- $i$ minor of $G$ obtained by contracting the disjoint $i$-neighbourhoods of vertices in $B_{i}^{\prime}$. Let $C$ be a $\Delta_{f(i+1)}$-indiscernible subsequence of $\tilde{B}_{i}^{\prime}$ in $G_{i}^{\prime}$. Define $S_{i+1}$ as in case $i=1$, i.e. as the set of vertices $v$ with $C \subseteq N(v)$. Note that we constructed $G_{i}^{\prime}$ and did not work with $H$ because otherwise the vertices of $S_{i+1}$ could have been contracted 
vertices. Those vertices are subgraphs of $G$ and can be arbitrarily large. Hence we would possibly delete much more than $\left|S_{i+1}\right|$ vertices.

Define $B_{i+1} \subseteq B_{i}^{\prime}$ as the set of vertices with $C=\left\{G\left[N_{i}^{G-Z_{i}}(v)\right]: v \in B_{i+1}\right\}$. If $C$ is large enough (just as in the case $i=1$ ), $C$ is 2 -independent in $G_{i}^{\prime}-Z_{i+1}$, so $B_{i+1}$ is $2(i+1)$-independent in $G-Z_{i+1}$.

For $i=r$ we are left with a set of size $m$ if we started with a set of size $p_{r}(m)$ with $p_{r}(x)$ chosen appropriately, tracing back the construction. The vertices $Z_{i}$ we delete during the construction are connected with all (contracted) vertices $\tilde{B}_{i}$. Note that the vertices of $Z_{r}$ we delete are connected to vertices at distance at most $r-1$ to the vertices of $B_{r}$. We can hence merge the vertices $z$ of $Z_{r}$ with the first $\left|Z_{r}\right|$ branch sets $\tilde{B}_{r}$ (each vertex $z$ with one distinct branch set) to build a complete depth- $r$ minor of order $\left|Z_{r}\right|$. Hence we can take $s(r):=f(r)$, as this bounds the size of a largest complete depth- $r$ minor by assumption.

We now show how to compute the sets $B_{i}, \tilde{B}_{i}$ and $Z_{i}$. In iteration $i$ we want to compute a $\Delta_{f(i)}$-indiscernible sequence as in the proof of Theorem 15. We remark that usually a graph $G$ from a nowhere dense class will be stored as a list of adjacency lists. As, for every $\varepsilon>0, G$ is $c$-degenerate for some $c \in \mathcal{O}\left(n^{\varepsilon}\right)$, we can store the adjacency relation in a more efficient way. Let $L$ be a linear order such that for each $v \in V(G)$ we have $\left|\left\{w \in N(v): w<_{L} v\right\}\right| \leq c$, i.e. every vertex has at most $c$ smaller neighbours. Now for every vertex we store the set of its smaller neighbours. Then we can implement an adjacency test in time $\mathcal{O}\left(n^{\varepsilon}\right)$ for any fixed $\varepsilon>0$. Model-checking for the formulas of $\Delta_{i}$ with one quantifier is hence possible in time $\mathcal{O}\left(n^{1+\varepsilon}\right)$ by Theorem 15 . Furthermore, we have to perform the first $r$ levels of breadth-first searches around the elements of $A$ to keep track of the sets $\bar{B}_{i}$. Note that the $i$-neighbourhoods of these elements are disjoint when the searches are taken one step further (after deleting the set $S_{i}$ ). Hence, every edge appears at most once in all searches, and the searches can be carried out in time $\mathcal{O}\left(n^{1+\varepsilon}\right)$. Here we use again that a sufficiently large graph from a nowhere dense class of graphs has at most $n^{1+\varepsilon}$ edges for any fixed $\varepsilon>0$.

\section{A Polynomial Kernel for Distance- $r$ Dominating Set}

We now show how to obtain a polynomial kernel for the Distance- $r$ Dominating Set problem. Recall that a kernelization algorithm, or short kernel, for the DisTANCE- $r$ Dominating SET problem parameterized by the solution size is a polynomial time algorithm, which on input $G$ and $k$ computes another graph $H$ and a new parameter $k^{\prime}$ which is bounded by a function of $k$ (independent of $|V(G)|$ ), such that $G$ contains a distance- $r$ dominating set of size at most $k$ if, and only if, $H$ contains a distance- $r$ dominating set of size at most $k^{\prime}$. By abuse of notation, we also call the output of a kernelization algorithm a kernel. If $|V(H)|$ is bounded by a polynomial in $k$, then the kernel is called a polynomial kernel. We remark that by our definition, a kernelization algorithm on a class $\mathcal{C}$ does not necessarily output graphs from $\mathcal{C}$. Very strictly speaking, we are hence computing a bi-kernel and not a kernel (as we reduce not to an instance of the original problem "distance- $r$ dominating set on $\mathcal{C}$ " but to the formally different problem "distance-r dominating set).

The idea is to kernelise the instance in two phases. In the first phase we reduce the number of dominatees, that is, the number of those vertices whose domination is essential. More precisely, for an integer $k$, a set $Z \subseteq V(G)$ is called an $r$-domination core for parameter $k$ if every set $X \subseteq V(G)$ of size at most $k$ which $r$-dominates $Z$ also $r$-dominates $V(G)$. In the second phase we reduce the number of dominators, that is, the number of vertices that shall be used to dominate other vertices. 
For the first phase, we argue just as Dawar and Kreutzer in Lemma 11 of [5], to obtain an $r$-domination core in $G$. The key idea of the lemma is to find a large $2 r$-independent set $A$ after deleting at most $s$ vertices, such that at least two elements of $A$ behave alike with respect to every small dominating set.

Fix a nowhere dense class $\mathcal{C}$ of graphs and let $N(m, r)=p_{r}(m)$ for the polynomial $p_{r}(x)$ and $s(r)$ characterising $\mathcal{C}$ as uniformly quasi-wide according to Theorem 4. Fix positive integers $r$ and $k$ and let $s:=s(2 r)$. Let $c$ be the minimum integer such that $K_{c} \aleph_{2 r} G$ for all $G \in \mathcal{C}$.

The proof of the following lemma is the same as in [5], we just use better bounds from Theorem 4 .

Lemma 20 (see [5], Lemma 11). For $r \geq 0$ let $p_{r}$ be the polynomial defined in Theorem 4. There is an algorithm that, given an n-vertex graph $G \in \mathcal{C}, \varepsilon>0, k>0$ and $Z \subseteq V(G)$ with $|Z|>$ $p_{2 r}\left((k+2)(2 r+1)^{s}\right)=: \ell$ runs in time $\mathcal{O}\left(s \cdot \ell^{c+1} \cdot r \cdot c \cdot n^{1+\varepsilon}\right)$, and returns a vertex $w \in Z$ such that for any set $X \subseteq V(G)$ with $|X| \leq k$,

\section{$X r$-dominates $Z$ if, and only if, $X r$-dominates $Z \backslash\{w\}$.}

Proof. Fix a set $A \subseteq Z$ of size exactly $\ell$. By Theorem 4 we can find in time $\mathcal{O}\left(r \cdot c \cdot \ell^{c+1} \cdot n^{1+\varepsilon}\right)$ sets $B \subseteq A$ and $S \subseteq V(G)$ such that $|S| \leq s$ and $|B| \geq(k+2)(2 r+1)^{s}$ such that $B$ is $2 r$-independent in $G-S$. Let $S=\left\{t_{1}, \ldots, t_{s}\right\}$ and, for each $v \in B$, compute the distance vector $d_{v}=\left(d_{1}, \ldots, d_{s}\right)$, where $d_{i}=\operatorname{dist}\left(v, t_{i}\right)$ if this distance is at most $2 r$ and $d_{i}=\infty$, otherwise. For this, we have to perform $s$ breadth-first searches which takes time $\mathcal{O}\left(s \cdot n^{1+\varepsilon}\right)$. Note that there are at most $(2 r+1)^{s}$ distinct distance vectors. Since $\ell \geq(k+2)(2 r+1)^{s}$, there are at least $k+2$ elements $b_{1}, \ldots, b_{k+2} \in B$ which have the same distance vector. Now we choose $w:=b_{1}$ and show that for any set $X \subseteq V(G)$ with $|X| \leq k, X r$-dominates $Z$ if, and only if, $X r$-dominates $Z \backslash\left\{b_{1}\right\}$.

The direction from left to right is obvious. Now, suppose $X r$-dominates $Z \backslash\left\{b_{1}\right\}$. Consider the sets $B_{i}:=N_{r}^{G-S}\left(b_{i}\right)$ for $i \in[2, \ldots, k+2]$. These sets are, by construction, mutually disjoint. Since there are $k+1$ of these sets, at least one of them, say $B_{j}$, does not contain any element of $X$. However, since $b_{j} \in Z \backslash\left\{b_{1}\right\}$ there is a path of length at most $r$ from some element $x$ in $X$ to $b_{j}$. This path must, therefore, go through an element of $S$. Since $b_{1}$ and $b_{j}$ have the same distance vector, we conclude that there is also a path of length at most $r$ from $x$ to $b_{1}$ and therefore $X$ $d$-dominates $Z$.

For the complexity bounds, note that all distance vectors can be computed in time $\mathcal{O}(|S| \cdot|A|$. $|G|)=\mathcal{O}\left(s \cdot p_{2 r}\left((k+2)(2 r+1)^{s}\right) \cdot n\right)$ (recall that $G$ is degenerate). Adding this to the $\mathcal{O}\left(r \cdot c \cdot \ell^{c+1} \cdot n^{1+\varepsilon}\right)$ time to find $S$ and $A$ gives us the required bound.

We now proceed as follows. We let $Z=V(G)$ and apply the above procedure to remove an irrelevant element from the $r$-domination core $Z$ until $|Z| \leq p_{2 r}\left((k+2)(2 r+1)^{s}\right)$.

Corollary 21. Let $\mathcal{C}$ be a nowhere dense class of graphs and let $k, r \in \mathbb{N}$. There is an algorithm running in time $\mathcal{O}\left(n^{2+\varepsilon}\right)$ that given an n-vertex graph $G \in \mathcal{C}$ and $\varepsilon>0$, computes an $r$-domination core for parameter $k$ of $G$ of size polynomial in $k$.

We now reduce the number of dominators, that is, the number of vertices that shall be used to dominate other vertices. For this, observe that only vertices at distance at most $r$ to a vertex from the $r$-domination core are relevant. Furthermore, if there are two vertices $u, v \in V(G)$ with $N_{r}(u) \cap Z=N_{r}(v) \cap Z$, it suffices to keep one of $u$ and $v$ as a representative. 
Lemma 22. Let $\mathcal{C}$ be a nowhere dense class of graphs and let $r \in \mathbb{N}$. There is an algorithm running in time $\mathcal{O}\left(n^{1+\varepsilon} \cdot|Z|^{1+\varepsilon}\right)$, that given an $n$-vertex graph $G \in \mathcal{C}, \varepsilon>0$ and $Z \subseteq V(G)$ computes a minimum size set $Y \subseteq V(G)$ such that for all $u \in V(G)$ there is $v \in Y$ with $N_{r}(u) \cap Z=N_{r}(v) \cap Z$.

Proof. First, each element $v \in V(G)$ learns the set $N_{r}(v) \cap Z$. For this, we perform $|Z|$ breadthfirst searches of depth $r$ starting at the elements of $Z$. Whenever a breadth-first search around $z \in Z$ encounters the element $v$, it registers the element $z$ in a list of $v$. For any $\varepsilon>0$, this takes time $\mathcal{O}\left(|Z| \cdot n^{1+\varepsilon}\right)$ on a graph from a nowhere dense class.

Now, every element $v \in V(G)$ orders its list containing the elements of $N_{r}(v) \cap Z$ in an increasing order. We assume here, that every vertex is identified by a number between 1 and $n$. This takes time $\mathcal{O}(n \cdot|Z| \cdot \log |Z|)$. For two elements $v, w \in V(G)$, we can now decide whether $N_{r}(v) \cap Z=N_{r}(w) \cap Z$ in time $\mathcal{O}(|Z|)$ by comparing the ordered lists representing their neighbourhood intersections.

We now order the vertices of $V(G)$ according to their neighbourhood intersections in time $\mathcal{O}(n \cdot \log n \cdot|Z|)$. We remove duplicates from the sorted list in time $\mathcal{O}(n \cdot|Z|)$ to output the set $Y$.

Note that $\log n \leq n^{\varepsilon}$ for all fixed $\varepsilon>0$ and sufficiently large $n$. By rescaling $\varepsilon$, we get a total running time of $\mathcal{O}\left(n^{1+\varepsilon} \cdot|Z|^{1+\varepsilon}\right)$.

According to Corollary 14, the set $Y$ we compute in Lemma 22 has size at most $|Z|^{s}$ for some integer $s$ depending only on $r$ and $\mathcal{C}$.

Let $Z$ be a $r$-domination core of $G$, let $Y \subseteq V(G)$ be a minimum size set such that for all $u \in V(G)$ there is $v \in Y$ with $N_{r}(u) \cap Z=N_{r}(v) \cap Z$. We construct a graph $H$ whose vertex set is the union of $Z$ and $Y$. For every $v \in Y$, compute $N_{r}(v) \cap Z$ and add the vertices and edges of a shortest path (of length at most $r$ ) between $v$ and each $z \in N_{r}(v) \cap Z$ to $H$, such that $N_{r}^{G}(v) \cap Z=N_{r}^{H}(v) \cap Z$. As $\left|N_{r}^{G}(v) \cap Z\right|$ is bounded by $|Z|$, we conclude that $|V(H)| \leq r \cdot|Z|^{s+1}$. We now add two new vertices $v, v^{\prime}$ to $H$ and connect $v$ to every vertex except to the vertices of $Z$ by a path of length $r$ and to $v^{\prime}$ by a path of length $r$. It is easy to see (compare to Lemma 2.16 of [8]) that there exists a set $D$ of size $k$ which $r$-dominates $Z$ if, and only if, $H$ admits an distance- $r$ dominating set of size $k+1$. Hence, the size of $H$ is polynomially bounded by $k$ and $H$ can be computed in polynomial time by combining the above lemmas. This proves the main theorem of this section.

Theorem 23. Let $\mathcal{C}$ be a nowhere dense class of graphs. For every $r \in \mathbb{N}$ there is a polynomial $p(x)$, a constant $c$ and an algorithm running in time $\mathcal{O}\left(p(k)^{c} \cdot n^{2+\varepsilon}\right)$ which, given a graph $G \in \mathcal{C}, \varepsilon>0$ and $k \in \mathbb{N}$ computes a kernel for the Distance- $r$ Dominating Set problem of size $p(k)$ on $G \in \mathcal{C}$.

\section{Single Exponential Parameterized Algorithms on Nowhere Dense Classes}

In [5], the authors show how the concept of uniform quasi-wideness can be used to design parameterized algorithms for dominating set problems on nowhere dense classes of graphs. However, the dependence on the parameter in the algorithms in [5] is enormous, usually manyfold exponential.

In this section we combine the tools developed in the previous section with the general technique for obtaining parameterized algorithms in [5] to design parameterized algorithms for several graph problems on nowhere dense classes with only a single exponential dependence on the parameter value. 
We demonstrate the idea by showing that the Connected Dominating Set problem can be solved in time $2^{p(k)} \cdot n^{c}$ for a fixed constant $c$ and a polynomial $p(x)$. This example is particularly interesting as it was shown in [9] that the problem is unlikely to have a polynomial kernel on nowhere dense classes. Hence a single exponential parameter dependence cannot be obtained from a polynomial kernelization algorithm.

Theorem 24. Let $\mathcal{C}$ be a nowhere dense class of graphs. Then there is a polynomial $p(x)$, and an algorithm running in time $\mathcal{O}\left(2^{p(k)} \cdot n^{1+\varepsilon}\right)$ which, given an n-vertex graph $G, \varepsilon>0$ and a number $k$ as input, decides whether $G$ contains a connected dominating set of size $k$.

Proof. For $i \geq 1$ let $d_{i}$ be the minimum size of a clique that cannot be obtained as depth- $i$ minor in any member $H \in \mathcal{C}$. Let $s$ and $N$ be a margin of the class $\mathcal{C}$. By Theorem 4 , we can choose $s(r)$ such that $s(r) \leq d_{r}$ and $N(r, m) \leq m^{c(r)}$ for some function $c(r)$ depending only on $r$.

Let $G$ and $k$ be given. Let $s:=s(1)=d_{1}$ and let $K:=N(1, k+1)=(k+1)^{c(1)}$. During the algorithm we will maintain sets $W_{i}$ and $X_{i}$ of vertices such that $\left|X_{i}\right|=i$ and $W_{i}$ is the set of vertices in $G$ not dominated by any member of $X_{i}$. We initialise $W_{0}:=V(G)$ and $X_{0}:=\emptyset$.

Now, after $i$ steps, suppose that $W_{i}, X_{i}$ have been defined. If $\left|W_{i}\right| \geq K$, then we use Theorem 4 to compute a set $S$ of size $|S| \leq s$ and a set $A \subseteq W$ of size $k+1$ such that $A$ is 2-independent in $G-S$. As $A$ is 2 -independent, no vertex in $G-S$ can dominate two members of $A$. Hence, if there is a connected dominating set in $G$ using the vertices in $X_{i}$, then this set will need to include an element of $S$. We now branch over the $s$ possible choices of an element in $S$ and for each such $v \in S$ call the algorithm recursively with sets $X_{i+1}:=X_{i} \cup\{v\}$ and $W_{i+1}:=W_{i} \backslash N[v]$, where $N[v]$ denotes the closed neighbourhood of $v$, i.e. $N[v]:=N(v) \cup\{v\}$.

If $i=k$, the recursion stops. If $W \neq \emptyset$, then we can return "no" as there cannot be any connected dominating set of size at most $k$ using the vertices in $X_{i}$. If $W_{i}=\emptyset$, then we found a solution if, and only if, $X_{i}$ is connected.

Otherwise we have $i<k$ and the recursion stops because $\left|W_{i}\right|<K$. We now have a set $X_{i}$ of $\left|X_{i}\right|=i<k$ vertices for our dominating set and still need to dominate $W_{i}$. Furthermore, we still need to connect the dominating set.

We suppose that $\ell \leq k-i$ vertices $y_{1}, \ldots, y_{\ell}$ are used to dominate $W_{i}$ and at most $k-i-\ell$ further vertices to connect the dominating set $X_{i} \cup\left\{y_{1}, \ldots, y_{\ell}\right\}$. For every $y_{j}$ we guess a set $Y_{j}$ that is dominated by $y_{j}$ and such that the sets $Y_{j}$ form a partition of $W_{i}$. We do not forbid that a vertex $y_{j}$ also dominates some vertices in a $Y_{j^{\prime}}$ for $j \neq j^{\prime}$. Here the $Y_{j}$ 's are guessed and the $y_{j}$ 's are then computed. In other words, for any partition of $W_{i}$ into $l \leq k-i$ non-empty sets $Y_{1}, \ldots Y_{l}$ we do the following. First, for each $Y_{j}$ we compute the set $D_{j}$ of vertices $v \in V(G)$ such that $Y_{j} \subseteq N[v]$. If for some $j$ there is no such vertex, then we discard this partition. So suppose $Y_{1}, \ldots, Y_{l}$ is a partition such that $D_{1}, \ldots, D_{l}$ are all non-empty. Then we can define a dominating set of $G$ by adding one element $w_{j}$ of each $D_{j}$ to the set $X_{i}$. By construction, every vertex $v \in V(G) \backslash W_{i}$ is dominated by a member of $X_{i}$ and every $v \in W_{i}$ is dominated by the vertex $w_{j}$ chosen for the partition $Y_{j}$ containing $v$.

Hence, all that remains is to show that we can choose the $w_{1}, \ldots, w_{l}$ so that $X_{i} \cup\left\{w_{1}, \ldots, w_{l}\right\}$ can be turned into a connected set by adding at most $k-(i+l)$ extra vertices. We solve this problem by using the Dreyfus-Wagner algorithm [10] for solving Steiner trees. The Dreyfus-Wagner algorithm computes a minimum Steiner tree for a set of at most $T$ terminals in $\mathcal{O}\left(3^{T} n+2^{T} n^{2}+n^{2} \log n+n m\right)$ time, where $m$ is the number of edges of the input graph on $n$ vertices.

Now, suppose we are given the set $X_{i}$ of size $i$, the partition $Y_{1}, \ldots, Y_{l}$ of $W_{i}$ into disjoint sets, for $l \leq k-i$ and the sets $D_{1}, \ldots, D_{l}$. For all $1 \leq j \leq l$ we add a fresh vertex $t_{j}$ to the graph $G$ and 
add a path $P\left(t_{j}, v\right)$ of length 3 between $t_{j}$ and every member $v$ of $D_{j}$ (so that the paths $P\left(t_{j}, v\right.$ ) and $P\left(t_{j^{\prime}}, v^{\prime}\right)$ are internally vertex disjoint if $\left.\left\{t_{j}, v\right\} \neq\left\{t_{j^{\prime}}, v^{\prime}\right\}\right)$. Let $G^{\prime}$ be the augmented graph. We now call the Dreyfus-Wagner algorithm on $G^{\prime}$ with terminal set $X_{i} \cup\left\{t_{1}, \ldots, t_{l}\right\}$. Let $T$ be the resulting Steiner tree. Then $T$ needs to contain at least one vertex of every set $D_{j}$, as this is the only way to connect the terminal $t_{j}$ to the rest of the graph. Hence, any Steiner tree $T$ is a connected dominating set of $G^{\prime}$.

Note that in any minimum size Steiner tree for this terminal set, every $t_{j}$ is connected by exactly one path $P\left(t_{j}, v\right)$ to a vertex $v \in D_{j}$. To see this, recall that every $w \in Y_{j}$ has every vertex in $D_{j}$ as its neighbour. The only reason why a minimum Steiner tree might contain two paths $P\left(t_{j}, v\right)$ and $P\left(t_{j}, v^{\prime}\right)$ for distinct $v, v^{\prime} \in D_{j}$ is that this is needed to connect $v$ and $v^{\prime}$. But then, the path $P\left(t_{j}, v^{\prime}\right)$ can be replaced by adding any member of $Y_{j}$ instead. As $P\left(t_{j}, v^{\prime}\right)$ has two internal vertices, this would decrease the size of the Steiner tree. In particular this implies that every vertex $t_{j}$ is a leaf of a minimum size Steiner tree.

We can now turn $T$ into a connected dominating set for $G$ as follows. We simply delete for each $t_{j}$ the vertex $t_{j}$ and all internal vertices of the (unique) path $P\left(t_{j}, v\right)$ connecting $t_{j}$ to a member of $D_{j}$. By the argument above, the resulting tree $T^{\prime}$ is still connected and forms a connected dominating set of $G$. Conversely, any minimum size connected dominating set for $G$ containing $X_{i}$ and also at least one vertex of each $D_{j}$ can be extended to a minimum size Steiner tree in $G^{\prime}$ with the terminal set as above. It follows that if for any partition $Y_{1}, \ldots, Y_{t}$ we obtain a Steiner tree as above of size at most $k$, then we can return this tree as a connected dominating set, and otherwise can conclude that there is no connected dominating set of size at most $k$ using the vertices in $X_{i}$ and the partition $Y_{1}, \ldots, Y_{\ell}$.

Hence, the whole search tree yields a correct decision procedure for the connected dominating set problem. The running time is bounded by the size of the search tree and the time the algorithm takes at each leaf of the search tree. Note that the branching of the search tree is bounded by $s=d_{1}$ and the depth by $k$. Hence, in total we have at most $s^{k+1}-1$ nodes. At every leaf we have to consider every possible partition of the set $W_{i}$ of size at most $K=(k+1)^{c(1)}$ into at most $k$ disjoint sets. For each partition we call the Dreyfus-Wagner algorithm whose running time is dominated by $\mathcal{O}\left(3^{k}\left|W_{i}\right|^{2} \cdot \log \left|W_{i}\right|\right)$. Hence, the total running time of the algorithm is bounded by $2^{p(k)} \cdot n^{1+\varepsilon}$ for a polynomial $p$.

The proof method used in the previous theorem can be used to establish single exponential parameterized algorithms for other problems on nowhere dense classes of graphs. Hence, while the general proof technique is the same, we can dramatically improve the running time of our algorithms from a multiple exponential parameter dependence to single exponential.

\section{Conclusion and recent results}

Nowhere dense classes are classes of uniformly sparse graphs with a rich algorithmic theory. Especially the characterisation of these classes via uniform quasi-wideness finds many algorithmic applications. In this paper we have proved polynomial bounds on the margin of uniformly quasiwide classes, and hence nowhere dense classes. These bounds yield new algorithmic tools, especially in the area of parameterized complexity for nowhere dense graph classes. We have derived a polynomial kernel for the Distance- $r$ Dominating Set problem and a parameterized algorithm with single exponential parameter dependence for the Connected Dominating Set problem. 
The polynomial bounds on the margin of uniformly quasi-wide classes are established by a connection to stability theory, a classical field of infinite model theory. The proof is based a nonconstructive argument of Adler and Adler [1], who observed that nowhere dense graph classes are stable. As a consequence, our upper bounds are given purely existentially and are not effectively computable. A purely combinatorial and effective proof has recently been given in [27]. Lower bounds, showing that polynomial bounds are the best one can hope for even on classes of graphs which exclude a fixed minor, were established in [21].

The kernelization results for the Distance- $r$ Dominating Set problem have recently been improved from polynomial kernels to almost linear kernels in [12]. The ConneCted Dominating SET problem does not admit polynomial kernels even on bounded expansion classes of graphs (unless $\mathrm{NP} \subseteq$ coNP/poly) [9]. However, lossy kernels of linear size for the Connected Dominating SET problem on classes of bounded expansion and lossy kernels of polynomial size for the CONNECTED Distance-r Dominating SeT problem on nowhere dense classes were recently established in [11].

\section{References}

[1] Hans Adler and Isolde Adler. Interpreting nowhere dense graph classes as a classical notion of model theory. European Journal of Combinatorics, Vol. 36:322-330, 2014.

[2] Jochen Alber, Michael R. Fellows, and Rolf Niedermeier. Polynomial-time data reduction for dominating set. Journal of the ACM (JACM), 51(3):363-384, 2004.

[3] Hans L. Bodlaender, Fedor V. Fomin, Daniel Lokshtanov, Eelko Penninkx, Saket Saurabh, and Dimitrios M Thilikos. (Meta) kernelization. In Foundations of Computer Science, 2009. FOCS'09. 50th Annual IEEE Symposium on, pages 629-638. IEEE, 2009.

[4] Anuj Dawar. Homomorphism preservation on quasi-wide classes. Journal of Computer and System Sciences, 76(5):324-332, 2010.

[5] Anuj Dawar and Stephan Kreutzer. Domination problems in nowhere-dense classes. In IARCS Annual Conference on Foundations of Software Technology and Theoretical Computer Science, FSTTCS, pages 157-168, 2009.

[6] Erik D. Demaine and MohammadTaghi Hajiaghayi. Equivalence of local treewidth and linear local treewidth and its algorithmic applications. In Proceedings of the fifteenth annual ACMSIAM symposium on Discrete algorithms, pages 840-849. Society for Industrial and Applied Mathematics, 2004.

[7] Reinhard Diestel. Graph Theory: Springer Graduate Text GTM 173, volume 173. Reinhard Diestel, 2012.

[8] Pål Grønås Drange, Markus S. Dregi, Fedor V. Fomin, Stephan Kreutzer, Daniel Lokshtanov, Marcin Pilipczuk, Michał Pilipczuk, Felix Reidl, Saket Saurabh, Fernando Sánchez Villaamil, Saket Saurabh, Sebastian Siebertz, and Somnath Sikdar. Kernelization and sparseness: the case of dominating set. arXiv:1411.4575, 2015.

[9] Pål Grønås Drange, Markus Sortland Dregi, Fedor V. Fomin, Stephan Kreutzer, Daniel Lokshtanov, Marcin Pilipczuk, Michał Pilipczuk, Felix Reidl, Fernando Sanchez Villaamil, Saket 
Saurabh, Sebastian Siebertz, and Somnath Sikdar. Kernelization and sparseness: the case of dominating set. In 33rd Symposium on Theoretical Aspects of Computer Science, STACS, pages 31:1-31:14, 2016.

[10] S. E. Dreyfus and R. A. Wagner. The steiner problem in graphs. Networks, 1:195-207, 1972.

[11] Eduard Eiben, Mithilesh Kumar, Amer E. Mouawad, Fahad Panolan, and Sebastian Siebertz. Lossy kernels for connected dominating set on sparse graphs. In 35th Symposium on Theoretical Aspects of Computer Science, STACS, pages 29:1-29:15, 2018.

[12] Kord Eickmeyer, Archontia C. Giannopoulou, Stephan Kreutzer, O-joung Kwon, Michal Pilipczuk, Roman Rabinovich, and Sebastian Siebertz. Neighborhood complexity and kernelization for nowhere dense classes of graphs. In 44th International Colloquium on Automata, Languages, and Programming, ICALP, pages 63:1-63:14, 2017.

[13] Fedor V. Fomin, Daniel Lokshtanov, Saket Saurabh, and Dimitrios M Thilikos. Bidimensionality and kernels. In Proceedings of the twenty-first annual ACM-SIAM symposium on Discrete Algorithms, pages 503-510. Society for Industrial and Applied Mathematics, 2010.

[14] Fedor V. Fomin, Daniel Lokshtanov, Saket Saurabh, and Dimitrios M Thilikos. Linear kernels for (connected) dominating set on H-minor-free graphs. In Proceedings of the twenty-third annual ACM-SIAM symposium on Discrete Algorithms, pages 82-93. SIAM, 2012.

[15] Fedor V. Fomin, Daniel Lokshtanov, Saket Saurabh, and Dimitrios M. Thilikos. Linear kernels for (connected) dominating set on graphs with excluded topological subgraphs. In 30th International Symposium on Theoretical Aspects of Computer Science, STACS, pages 92-103, 2013.

[16] Jakub Gajarskỳ, Petr Hliněnỳ, Jan Obdržálek, Sebastian Ordyniak, Felix Reidl, Peter Rossmanith, Fernando Sánchez Villaamil, and Somnath Sikdar. Kernelization using structural parameters on sparse graph classes. Journal of Computer and System Sciences, 84:219-242, 2017.

[17] Martin Grohe, Stephan Kreutzer, and Sebastian Siebertz. Deciding first-order properties of nowhere dense graphs. In Proceedings of the 46th Annual ACM Symposium on Theory of Computing, pages 89-98. ACM, 2014.

[18] Martin Grohe and Dániel Marx. Structure theorem and isomorphism test for graphs with excluded topological subgraphs. SIAM Journal on Computing, 44(1):114-159, 2015.

[19] Wilfrid Hodges. Model theory, volume 42. Cambridge University Press, 1993.

[20] Maryanthe Malliaris and Saharon Shelah. Regularity lemmas for stable graphs. Transactions of the American Mathematical Society, 366(3):1551-1585, 2014.

[21] Wojciech Nadara, Marcin Pilipczuk, Roman Rabinovich, Felix Reidl, and Sebastian Siebertz. Empirical evaluation of approximation algorithms for generalized graph coloring and uniform quasi-wideness. In 17th International Symposium on Experimental Algorithms, SEA, pages 14:1-14:16, 2018. 
[22] Jaroslav Nešetřil and Patrice Ossona de Mendez. Grad and classes with bounded expansion I. Decompositions. European Journal of Combinatorics, 29(3):760-776, 2008.

[23] Jaroslav Nešetřil and Patrice Ossona de Mendez. Grad and classes with bounded expansion II. Algorithmic aspects. European Journal of Combinatorics, 29(3):777-791, 2008.

[24] Jaroslav Nešetřil and Patrice Ossona de Mendez. First order properties on nowhere dense structures. The Journal of Symbolic Logic, 75(03):868-887, 2010.

[25] Jaroslav Nešetřil and Patrice Ossona de Mendez. On nowhere dense graphs. European Journal of Combinatorics, 32(4):600-617, 2011.

[26] Jaroslav Nešetřil and Patrice Ossona de Mendez. Sparsity. Springer, 2012.

[27] Michał Pilipczuk, Sebastian Siebertz, and Szymon Toruńczyk. On the number of types in sparse graphs. In Proceedings of the 33rd Annual ACM/IEEE Symposium on Logic in Computer Science, pages 799-808. ACM, 2018.

[28] Klaus-Peter Podewski and Martin Ziegler. Stable graphs. Fund. Math, 100(2):101-107, 1978.

[29] Neil Robertson and Paul D. Seymour. Graph minors. XVI. Excluding a non-planar graph. Journal of Combinatorial Theory, Series B, 89(1):43-76, 2003.

[30] Norbert Sauer. On the density of families of sets. Journal of Combinatorial Theory, Series A, 13(1):145-147, 1972.

[31] Saharon Shelah. A combinatorial problem; stability and order for models and theories in infinitary languages. Pacific Journal of Mathematics, 41(1):247-261, 1972.

[32] Saharon Shelah. Classification theory: and the number of non-isomorphic models, volume 92. Elsevier, 1990.

[33] Vladimir N. Vapnik and Alexey Y. Chervonenkis. On the uniform convergence of relative frequencies of events to their probabilities. In Measures of Complexity, pages 11-30. Springer, 2015. 